\title{
Growth and puberty in children with juvenile idiopathic arthritis
}

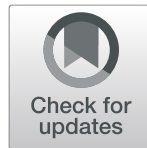

\author{
Debora Mariarita d'Angelo ${ }^{\dagger}$, Giulia Di Donato ${ }^{\dagger}$, Luciana Breda and Francesco Chiarelli ${ }^{*}$ (D)
}

\begin{abstract}
Juvenile Idiopathic Arthritis is one of the most prevalent chronic diseases in children, with an annual incidence of 2-20 cases per 100,000 and a prevalence of 16-150 per 100,000. It is associated with several complications that can cause short-term or long-term disability and reduce the quality of life. Among these, growth and pubertal disorders play an important role. Chronic inflammatory conditions are often associated with growth failure ranging from slight decrease in height velocity to severe forms of short stature. The prevalence of short stature in JIA varies from $10.4 \%$ in children with polyarticular disease to $41 \%$ of patients with the systemic form, while oligoarthritis is mostly associated with localized excessive bone growth of the affected limb, leading to limb dissymmetry. The pathogenesis of growth disorders is multifactorial and includes the role of chronic inflammation, long-term use of corticosteroids, undernutrition, altered body composition, delay of pubertal onset or slow pubertal progression. These factors can exert a systemic effect on the GH/IGF-1 axis and on the GnRH-gonadotropin-gonadic axis, or a local influence on the growth plate homeostasis and function. Although new therapeutic options are available to control inflammation, there are still $10-20 \%$ of patients with severe forms of the disease who show continuous growth impairment, ending in a short final stature. Moreover, delayed puberty is associated with a reduction in the peak bone mass with the possibility of concomitant or future bone fragility. Monitoring of puberty and bone health is essential for a complete health assessment of adolescents with JIA. In these patients, an assessment of the pubertal stage every 6 months from the age of 9 years is recommended. Also, linear growth should be always evaluated considering the patient's bone age. The impact of rhGH therapy in children with JIA is still unclear, but it has been shown that if rhGH is added at high dose in a low-inflammatory condition, post steroids and on biologic therapy, it is able to favor a prepubertal growth acceleration, comparable with the catch-up growth response in $\mathrm{GH}$-deficient patients. Here we provide a comprehensive review of the pathogenesis of puberty and growth disorders in children with JIA, which can help the pediatrician to properly and timely assess the presence of growth and pubertal disorders in JIA patients.
\end{abstract}

Keywords: Juvenile idiopathic arthritis, Puberty, Growth, Bone, Hormone replacement therapy

\footnotetext{
*Correspondence: chiarelli@unich.it

${ }^{\dagger}$ Debora Mariarita d'Angelo and Giulia Di Donato contributed equally to this work.

Department of Pediatrics, University of Chieti, Chieti, Italy
}

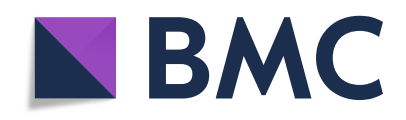

(c) The Author(s). 2021 Open Access This article is licensed under a Creative Commons Attribution 4.0 International License, which permits use, sharing, adaptation, distribution and reproduction in any medium or format, as long as you give appropriate credit to the original author(s) and the source, provide a link to the Creative Commons licence, and indicate if changes were made. The images or other third party material in this article are included in the article's Creative Commons licence, unless indicated otherwise in a credit line to the material. If material is not included in the article's Creative Commons licence and your intended use is not permitted by statutory regulation or exceeds the permitted use, you will need to obtain permission directly from the copyright holder. To view a copy of this licence, visit http://creativecommons.org/licenses/by/4.0/. The Creative Commons Public Domain Dedication waiver (http://creativecommons.org/publicdomain/zero/1.0/) applies to the data made available in this article, unless otherwise stated in a credit line to the data. 


\section{Background}

Juvenile Idiopathic Arthritis (JIA) is one of the most prevalent chronic diseases in children. It encompasses a heterogeneous group of arthritis of unknown origin beginning before the 16th birthday and lasting more than 6 weeks [1]. It is estimated that JIA has an annual incidence of 2-20 cases per 100,000 and a prevalence of 16-150 per 100,000 in high-income countries [2]. The etiology is still not completely elucidated, but it seems to be linked to a combination of genetic and environmental factors [1]. According to the International League of Associations for Rheumatology Classification (ILAR), seven subtypes of JIA have been identified, which differ for age and modality of presentation, clinical signs and symptoms, genetic background and prognosis: systemic JIA (sJIA), oligoarthritis, rheumatoid factor (RF)-positive and negative polyarthritis, psoriasic arthritis (PsA), enthesitis-related arthritis and undifferentiated arthritis [3]. Each subgroup of disease is associated with various degrees of severity and complications that can cause short-term or long-term disability and reduce the quality of life. Among these, growth and pubertal disorders play an important role. Thus, the aim of this paper is to supply a full review of the growth and pubertal disorders associated with JIA and to guide the pediatric rheumatologists and the general physicians in the follow up of these patients, in order to timely identify and properly treat children with growth and pubertal alterations.

\section{Growth and JIA}

Chronic inflammatory conditions are often associated with growth failure ranging from slight decrease in height velocity to severe forms of short stature. The prevalence of short stature varies from $10.4 \%$ in children with polyarticular disease to $41 \%$ of patients with the systemic form [4]. Oligoarthritis is mostly associated with localized excessive bone growth of the affected limb, with premature fusion of epiphyses leading to limb shortening in the older child; early treatment with intraarticular glucocorticoids (GCs) helps to prevent the onset of limb dissymmetry [5]. Data from the Childhood Arthritis Prospective Study (CAPS) showed that a cohort of children with JIA experienced growth restriction over the first 3 years of disease. Although height was within the normal range at presentation, growth restriction, defined as change of height $\mathrm{z}$-score less than 0.5 , was observed in $39 \%$ of patients and occurred early at disease onset, being greater in patients with sJIA and PsA. Forty-one per-cent of patients with persistent oligoarthritis experienced moderate growth restriction, more important in children who underwent several intraarticular corticosteroid injections. Higher childhood health assessment questionnaire (CHAQ) scores, longer duration of symptoms and lower Body Mass Index (BMI) z-score, were significantly associated with lower height zscores, like was total time on oral or intravenous steroid treatment with decrease in height $\mathrm{z}$-score from baseline to 3 years. Surprisingly, lower height at baseline predicted improvement of vertical growth over the first 3 years of follow up [6]. Some authors used data from the Research in Arthritis in Canadian Children emphasizing Outcomes (ReACCh-Out) cohort to assess growth in 1147 children newly diagnosed with JIA from 2005 to 2010. They reported that children with the most frequent JIA categories (oligoarthritis, RF-negative polyarthritis, enthesitis-related arthritis and undifferentiated arthritis) grew and gained weight as healthy children, while about $10 \%$ of children with sJIA, uncontrolled disease and/or prolonged use of steroids had an increased risk of growth impairment. A minor, but measurable, risk of growth failure was seen in children with RF-positive polyarthritis and PsA [7].

\section{Systemic and local regulation of linear growth}

Linear bone growth occurs at the growth plate and in consequent to a process of chondrogenesis and remodeling of cartilage into bone tissue (a process named "endochondral ossification"). The growth hormone (GH) and the insulinlike growth factor 1 (IGF-1) are important stimulators of longitudinal bone growth. According to the "somatomedin hypothesis", GH exerts its activity through the liver-derived IGF-1, which regulates proliferation, differentiation and apoptosis of many cell types [8]. GH binds to two receptors (GHRs) on the cell surface, causing a dimerization process and activating a series of other signalling molecules, like phosphatidyl-inositol-3-kinase (PI3K), mitogen-activated protein kinases (MAPKs), insulin receptor substrates (IRSs), protein kinase $\mathrm{C}(\mathrm{PKC})$, signal transducer and activator of transcription (STAT) etc. The GH binding protein (GHBP) is proteolyzed from the cell surface receptor and regulates $\mathrm{GH}$ bioavailability. $\mathrm{GH}$ also acts directly at the growth plate level to recruit resting chondrocytes into the proliferative state, with a subsequent increase local IGF-1 production, which stimulates chondrocytes proliferation and hypertrophy with an autocrine/paracrine mechanism ("dual effector hypothesis"). At a systemic level, six IGF binding proteins (IGFBPs), Acid Labile Subunit (ALS) and the mannose-6-phosphate receptor (type 2 IGF-1 receptor) are the main regulators of IGF-1 bioavailability. In fact, many studies showed that the most important regulator of growth is the level of free IGF-1 (not just the total IGF-1), which is also influenced by its binding to regulatory proteins, above all the IGFBP-3 [9].

\section{Pathogenesis of growth disorders in JIA}

The pathogenesis of growth disorders is multifactorial and includes the role of chronic inflammation, longterm use of supra-physiological doses of corticosteroids, 
undernutrition, altered body composition with lean mass reduction, physical inactivity, delays of pubertal onset or slow pubertal progression [10]. Also, the degree, extent and duration of disease activity are important, like the age at onset of the disease. These factors can exert a systemic effect on GH-IGF-1 axis, or a local influence on the growth plate homeostasis and function [11]. Growth in children with JIA is also influenced by sex steroids, thyroid hormone, parathyroid hormone-related peptide, bone morphogenic proteins, transforming growth factors and vitamin D metabolites.

\section{Growth disorders in JIA and inflammation}

Inflammation is characterized by the activation of different immune cells with the production of cytokines, chemokines, interleukins (ILs) and interferons (IFN), activating bone reabsorption and inhibiting bone formation at a systemic and/or local level. GH and IGFs are the most important regulators of linear growth. Chronic inflammation can interfere with the function of $\mathrm{GH}$ IGF-1 axis by inducing relative GH insufficiency, GH/ IGF-1 resistance, downregulation of GH/IGF-1 receptors, dysregulation of IGFBPs and thus of IGF-1 bioavailability, impairment of local GH and IGF-1 signaling pathways and gene regulation [12]. Several studies demonstrated the role of pro-inflammatory cytokines in influencing growth in children with chronic diseases and in animal models: interleukin-6 (IL-6), interleukin-1 $\beta$ (IL-1 $\beta)$ and tumor necrosis factor- $\alpha$ (TNF $\alpha)$ have been reported to have a major role in JIA.

\section{JIA inflammatory cytokines: systemic effects on the GH- IGF1 axis}

Elevated levels of IL-6 are found in serum and in synovial fluid of patients with JIA and they are associated with disease activity and laboratory markers of severity [13]. These patients, have reduced levels of circulating IGF-1, with normal GH. De Benedetti et al. [14] studied the effects of IL- 6 on growth regulation using the neurospecific enolase (NSE)/hIL-6 transgenic murine model, that overexpresses IL-6: NSE/hIL-6 mice have normal liver IGF-1 production, reduced levels of 3 IGFBP-3 and increased IGFBP-3 proteolysis, resulting in a marked decrease of circulating $150-\mathrm{kDa}$ ternary complex, even in the presence of functional ALS. Low concentrations of IGFBP-3 are also found in sJIA, with a marked decrease in IGF-1 half-life and increased IGF-1 clearance. IGFBP3 is produced by Kupffer cells, which express IL- 6 receptor, thus IL-6 may directly affect IGFBP-3 production or increase its proteolysis through the stimulation of proteases secretion (catepsin B and L, metalloproteinases MMP) from various cell types [11]. Furthermore, IL-6 and other pro-inflammatory cytokines induce the suppressor of cytokine signaling (SOCS) proteins, which reduce and regulate $\mathrm{GH}$ signaling; this may explain the GH resistance observed in inflammatory diseases [15]. Pro-inflammatory cytokines may also alter growth plate function by inhibiting IGF-1 intracellular signaling. TNF$\alpha$, IL- $1 \beta$ and IL- 6 attenuate IGF-1-induced activation of both MAPK and PI3K pathways in chondrocytes [16]. In addition, TNF- $\alpha$ and IL-1 $\beta$ inhibit the IGF-1-mediated phosphorylation of insulin receptor substrate-1 (IRS-1) in myoblasts, but it is unknown if this also occurs in chondrocytes [17]. Finally, IL-1 $\beta$ over-expression reduces IGF1 and ALS plasma levels. In septic rats, hepatic expression of IGFBP-1 is increased, which reduces IGF-1 bioavailability: this effect is completely reversed by treatment with an IL-1 receptor antagonist [18].

\section{JIA inflammatory cytokines: local effects on the growth plate}

In addition to its systemic effects, IL- 6 also acts on the growth plate environment and function reducing cartilagineous nodule formation, with an effect on the early differentiation steps of chondrocytes precursors [19]; it also decreases the synthesis of type II and X collagen and aggrecan. IL-6 can activate janus kinase (JAK)/ STAT signaling leading to down-regulation of type II collagen, aggrecan and link protein transcription in the articular chondrocytes [20]. In vivo studies also showed that IL-6 over-expression increases osteoclastogenesis with augmented bone reabsorption and osteopenia, reduces osteoblastic activity, determines defective ossification and decreases mineral apposition rate [21]. JIA is characterized by important changes in the articular environment and the immune cell proliferation causes localized hypoxia and $\mathrm{pH}$ reduction, thus influencing osteoblast function and bone mineralization [22]. Cartilage oligomeric matrix protein (COMP) is produced by chondrocytes and synovial cells and it is a marker of chondrocytes turnover in the growth plate. Children with sJIA have decreased COMP serum levels during active phases of disease, and those levels significantly increase after treatment with the anti-IL-6 tocilizumab. Likewise, serum bone alkaline phosphatase (BAP) concentration, which is a marker of osteoblast activity, is reduced during active disease and increased during remission phases. The active inflammatory state also increases MMP-3 concentration, which is useful for predicting joint damage [23]. While IL-6 acts mainly via systemic mechanisms on $\mathrm{GH}$ function, other cytokines, like IL- $1 \beta$ and TNF- $\alpha$, act primarily on the growth plate with an additive effect. They decrease the width of the proliferating zone of growth cartilage and the rate of endochondral ossification, reducing chondrocytes proliferation and stimulating apoptosis of proliferative cells. A possible mechanism of the induction of apoptosis is the down-regulation of gene expression of Sox-9, a 
regulatory factor for chondrocyte differentiation and cartilage formation [24]. Some authors found that the exposure of murine ATDC5 chondrogenic cells and metatarsals to IL- $1 \beta$ and TNF- $\alpha$ caused a decreased expression of collagen types II and X and aggrecan [19]. This study also showed a restricted potential for recovery of chondrogenesis and longitudinal bone growth after prolonged exposure to pro-inflammatory cytokines, thus explaining greater growth impairment in children with longer periods of symptoms before diagnosis and appropriate therapy initiation [10]. The most important effects of pro-inflammatory cytokines on GH-IGF-1 axis and on the growth plate are summarised in Fig. 1.

\section{Growth disorders in JIA and therapy}

Patient with JIA, especially those with systemic disease, often need to be treated with systemic steroids for long periods. GCs can inhibit GH pulsatile release, reduce GH and IGF-1 receptors expression by chondrocytes and impair IGF-1 signaling at the growth plate, mainly via the PI3K pathway [25]. GCs also reduce chondrocyte proliferation and differentiation by disrupting cell cycle progression and promoting cell cycle exit [26]. Finally, they stimulate chondrocytes' autophagy and apoptosis, by altering the amount of pro-apoptotic members of the Bcl-2 family like Bax and Bid [27]. The decreased cell proliferation during GCs treatment preserves chondrocytes proliferative capacity; therefore, after GCs discontinuation, growth plate chondrocytes are less senescent and have a greater proliferative potential [26]. That is why, after remission, growth often accelerates beyond the normal growth rate for age, a phenomenon called "catch up growth" and observed in many growthretarding conditions, like Cushing's syndrome, celiac disease and hypothyroidism. However, catch up growth in children may not be complete after discontinuation of GC if inflammation is ongoing [10]. Skeletal development and growth are reversibly impaired during GC treatment, but significant short stature or deviation from the target height has been reported only in children treated with systemic steroids for more than 1 year [28]. Moreover, it is difficult to differentiate the impact of GC therapy and of uncontrolled inflammation on growth impairment, even because the severity of the disease often correlates with the likelihood of treatment with GCs. Steroid treatment has a double role: it can delay or

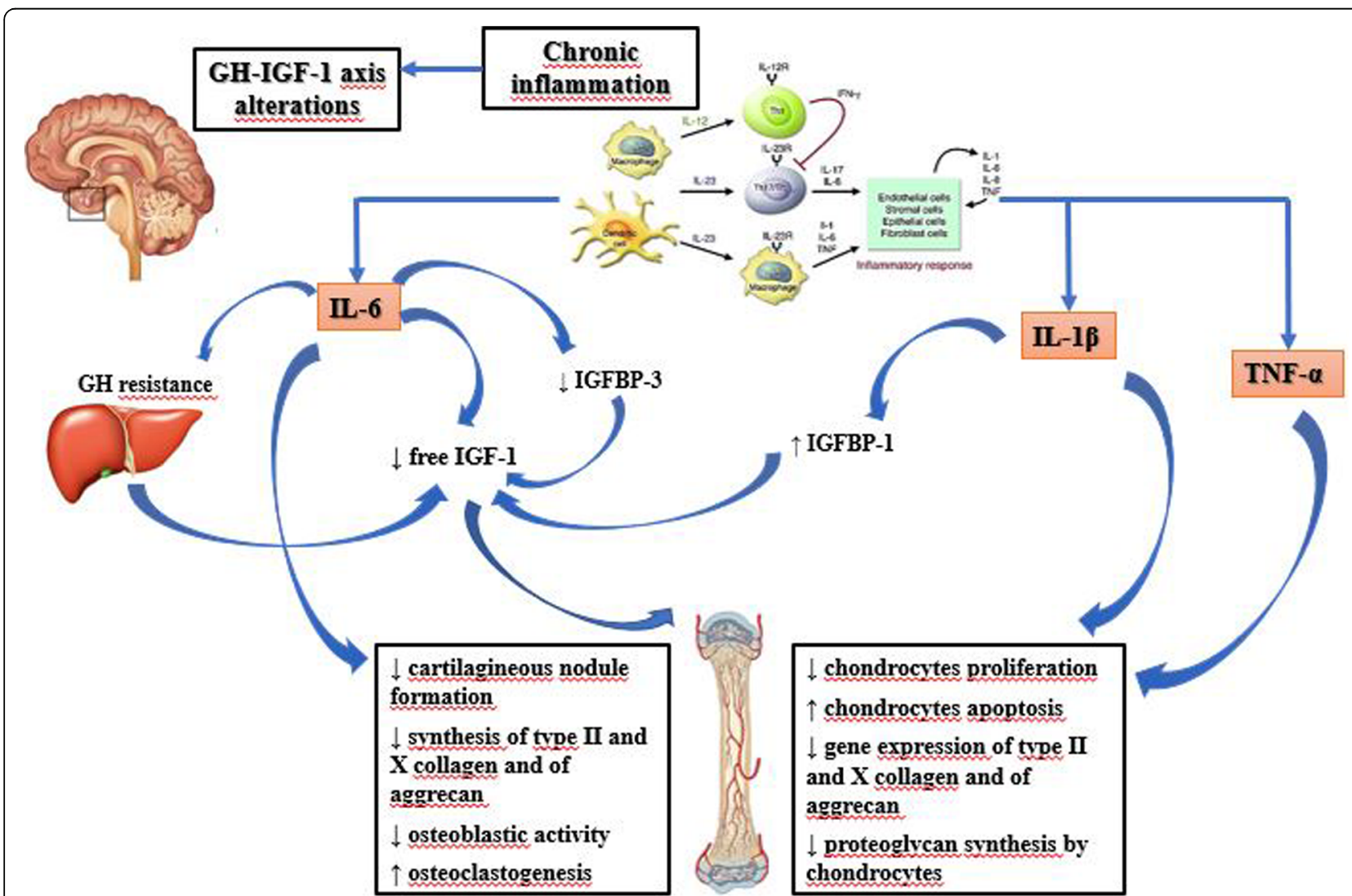

Fig. 1 Effects of pro- inflammatory cytokines on GH-IGF1 axis. GH (Growth Hormone); IGF-1 (Insulin-like Growth Factor-1); IGFBP-1 (Insulin-like Growth Factor Binding Protein-1); IGFBP-3 (Insulin-like Growth Factor Binding Protein-3); IL-6 (Interleukin-6); IL-1 $\beta$ (Interleukin-1 $\beta$ ); TNFa (Tumor Necrosis Factor-a) 
improve growth in patients with JIA, since its antiinflammatory effect may be positive especially in patients with SJIA, in whom active inflammation and high IL-6 levels may potentially affect growth [29]. Simon D. et al. retrospectively analyzed linear growth patterns during and after GC therapy in 24 prepubertal patients with sJIA, treated during at least the first 2 years (up to 7 years) with daily oral prednisone at a mean dosage of $0.2 \mathrm{mg} / \mathrm{kg}$ or more. Height data were expressed as the height standard deviation score for chronological age (HSDS/CA). A significant drop in height velocity (HV) (HSDS under $-2 \mathrm{SD}$ ) occurred during the first 4 years of the disease in $40 \%$ of patients and was correlated to the duration of prednisone therapy and with the severity of growth retardation during the active phase of the disease. Moreover, $70 \%$ of patients had a catch-up growth and $30 \%$ continued to show slow linear growth after prednisone discontinuation. Mean final height was below the target height in $80 \%$ of patients and was significantly correlated with mean height at prednisone discontinuation. These data suggest that early aggressive treatment increasing linear growth during the active phase of the disease could improve final height [4], and that aggressive control of inflammation, rather than GCs sparing, may be more important in the short-term to preserve normal growth [29]. On the other hand, the positive effect of steroid treatment needs to be balanced with the risk of long-term complications. In the last 10-15 years, immunomodulators and cytokines blockers have been introduced into routine clinical practice, managing to reduce the cumulative administered dose of GCs; furthermore, there is increasing use of intra-articular injections of GCs having less systemic side effects.

\section{Growth disorders in JIA and malnutrition}

The term "rheumatoid cachexia" has been used to describe the condition of decreased lean body mass, reduced energy expenditure and increased catabolism which is often associated with inflammatory diseases, like JIA [30]. In humans, malnutrition is associated with hepatic GH resistance with elevated systemic GH levels. Short periods of fasting cause elevation of fibroblast growth factor-21 (FGF-21), which regulates gluconeogenesis, fatty acids oxidation and ketogenesis: FGF-21 inhibits GH receptor binding in growth plate chondrocytes through the induction of leptin receptor overlapping transcript (LEPROT) and leptin receptor overlapping transcript-like 1 (LEPROTL1) activity [31].

\section{Management of growth failure in JIA}

Fighting the inflammatory process with immunomodulators (e.g., methotrexate), cytokine blockers (e.g., infliximab, etanercept, tocilizumab, anakinra), minimal effective use of GCs and optimal nutrition may improve growth, even if one-third of children with JIA continue to grow slowly and the reduction of disease activity does not seem to normalize linear growth in these patients [32].

Biologic treatments may reduce systemic inflammation and have a corticosteroid-sparing effect. Many studies have reported that anti-TNF treatments, the most widely used biologics, improve growth in patients with JIA [33]. Kearsley-Fleet L. et al. [34] evaluated the change over time in height z-score of 191 JIA patients treated with etanercept (ETN) for 2 years. Data about HV were compared with British median HV standards. The authors reported an improvement in mean height $\mathrm{z}$-score during the first 2 years of therapy (from -0.74 at the baseline to -0.45$)$, even if the cohort remained below the reference population mean, and patients with SIIA mantained the lowest $\mathrm{z}$-scores $(-1.45)$. Mean $\mathrm{HV}$ was $5.8 \mathrm{~cm} /$ year, greater than that of age- and gender-matched peers. The factors most strongly associated with the growth improvement were a lower height $\mathrm{z}$-score and no use of oral GCs at the baseline, even if patients receiving GCs at the start of ETN were mostly patients with sJIA. Moreover, data about patients' pubertal stage were missing, and the study did not demonstrate a significant association between changes in disease activity (Juvenile Arthritis Disease Activity Score-71 JADAS-71 or Minimal Disease Activity MDA score) and improved height z-score. Another study reported a mean growth improvement of $0.45 \mathrm{SD}$ after 2 years of therapy with antiTNF- $\alpha$ in 51 out of 71 children with JIA, with no significant efficacy in patients with severe JIA [35]. A further report of 52 patients treated with ETN showed that prepubertal children had no significant height gain [36]. Uettwiller et al. compared growth velocity in 100 prepubertal children (Tanner Stage <3) with JIA before and after the start of biologic therapy, continued for at least 6 months (mean duration of therapy 2.92 years) [32]. Patients were on follow up for 11 years on average. Fiftyone patients received anti-TNF $\alpha$ (ETN, infliximab, adalimumab), 12 were treated with the anti-IL-6 tocilizumab, 28 with anti-IL1 $\beta$ (canakinumab, anakinra), with the anti-CTLA-4 abatacept and 34 patients received more than one biologic drug. The authors did not show a significant difference between median HSDS at biologic treatment start and at the latest follow up, particularly in patients who received more than one biologic and in children with sJIA. In this study, the poor effect of biological therapy on growth could be due to the high proportion of patients with difficult-to-treat and sJIA. Nevertheless, $19 \%$ of patients remained below -2 SD or under their target height at the latest follow up: these data describe a better growth compared with patients not receiving biologics, whose final height is below -2SD in $41 \%$ of cases in sJIA and in $11 \%$ of cases in polyarticular JIA [37]. It can be concluded that biologic treatment 
can improve growth in patients with JIA but it seems insufficient to restore normal growth velocity, especially in systemic and severe forms of the disease. Early initiation of biologic therapy and selecting the optimal first biologic agent could help improving final height in patients with JIA [32].

Given the alterations of GH-IGF-1 axis associated with chronic inflammation, recombinant human GH (rhGH) therapy, especially at high doses, could be a therapeutic option in pre-pubertal children with chronic diseases and in patients with pubertal delay who continue to grow slowly [10]. Moreover, in children with severe JIA, the prevalence of $\mathrm{GH}$ deficit may be up to $18 \%$ [38]. Various studies explored the role of therapy with rhGH in patients with JIA and growth delay. Earlier retrospective and prospective studies reported that therapy with rhGH in patients with JIA can improve HV, producing an adult height similar to the target genetic potential. They also suggested a better growth response with higher doses [39-41]. Simon D. et al. [42] studied the effect of 3 years of therapy with rhGH at high dose $(0.46$ $\mathrm{mg} / \mathrm{kg} /$ week) given to 13 patients with JIA, evaluating the consequences on growth velocity, HSDS and body composition. They did not demonstrate significant changes of HSDS (from -4.6 at baseline to -4.3 at the latest follow up), but they showed an increase of lean mass by $33 \%$ and of lumbar bone mineral density (BMD) by $36.6 \%$, preventing further bone loss. Fat mass remained stable and only 6 patients developed transient glucose intolerance, without developing diabetes mellitus. Other studies similarly showed that rhGH therapy in JIA children can decrease insulin sensitivity, so that close monitoring of patients by oral glucose tolerance testing (OGTT) is crucial before and during rhGH treatment, particularly during puberty and disease relapses [43]. More recently, a number of randomized control trials (RCT) assessed the efficacy of rhGH therapy in JIA patients $[44,45]$. In a RCT of 31 children with JIA, patients treated with $\mathrm{GH}(0.33 \mathrm{mg} / \mathrm{kg} /$ week $)$ for 4 years had an increase in height of $1 \mathrm{SD}$, compared to the decrease of $0.7 \mathrm{SD}$ in untreated patients [46]. Long-term follow up data (7 years) indicate that mean final height was significantly greater in treated patients (SD $-1.6 \pm 0.25$ ) compared to control group (SD $-3.4 \pm 2$ ); besides, rhGH therapy did not affect age of puberty which was comparable between the two groups [38]. Simon D. et al. conducted an RCT study on a group of pre-pubertal children with recent JIA onset: after 3 years of therapy with $\mathrm{rhGH}(0.47 \mathrm{mg} / \mathrm{kg} /$ week $)$ they showed a relative height gain of $+1.5 \mathrm{SD}$ [47]. Small increase of lean mass, fat mass gain and lower bone mineral content (BMC) are often found in children with severe forms of JIA [48, 49]. Because of its anabolic effect, GH treatment, in these patients, causes reduction in fat mass and significant increase and normalization of total bone and muscle cross-sectional area (CSA) at final height [50].

There is now sufficient evidence to support that early use of relatively high dose of rhGH may improve linear growth in children with JIA. The acceleration in linear growth is associated with an increase of IGF-1 and IGFBP-3 serum levels, which persists during the continuation of therapy, showing a preserved GH-sensitivity [51]. However, catch up growth does not occur consistently and there is an important inter-individual variability in growth response [52]. The impact of therapy in patients with systemic and severe disease is still unclear. It seems that severe inflammation decreases the shortand long-term effect of rhGH therapy and that growth impairment during $\mathrm{GH}$ therapy correlates negatively with inflammatory markers and cumulative dose of GCs [53]. The role of pubertal stage should also be assessed, since the growth potential is also related to bone maturity: therefore, bone age should be considered, as a predictor for final HSDS.

\section{Puberty and JIA}

Puberty is a critical transition life process in which a complex series of hormonal and neurological changes result in the physical development of sexually mature adults [54]. The mechanisms governing the timing of puberty are currently a rich field of research and they are not completely understood. However, it is clear that many exogenous and endogenous factors (nutrition, genes, inflammatory status, endocrine-disruptor agents or societal changes) can influence the pubertal neuroendocrine events [55]. The evident plasticity of puberty timing and the importance of environmental factors allows adaptive changes that represent at the same time a limit and a resource for adolescent growth and health [56]. The activation and the progression of puberty are strong surrogate markers of adolescents' physical and mental health and this condition has obvious consequences in children with chronic diseases [56, 57]. Indeed, pubertal disorders in children with JIA, like in those with other inflammatory diseases, are frequently found, especially if they have prepubertal onset of disease and severe and prolonged chronic inflammatory course (sJIA) [58-60]. The most frequent pubertal abnormality described in these patients is delayed puberty, defined as the absence of testis enlargement or breast development at an age that is 2-2.5 SD later than the population mean (Tanner stage G2 in boys after the age of 14 years and B2 stage in girls after the age of 13 years in most Western countries) [61]. Slow clinical progression of puberty, isolated delayed menarche in females and decrease duration and intensity of puberty growth have also been reported [56]. However, few studies evaluating the prevalence of pubertal disorders, 
specifically in children with JIA, are available in the literature. Some authors found that the timing of menarche was later in all the 83 JIA patients in comparison with their mothers and normal Italian girls [58]. Similarly, other authors described a pubertal delay in 80 children with JIA compared to controls, less significant in the systemic onset JIA than in the oligoarticular and polyarticular groups [59]. In addition, other authors found a prevalence of delayed puberty of $15 \%$ in children with JIA, which is statistically significant compared to controls (1.4\%), and an association between dose of corticosteroids, age at the administration of corticosteroids and the delayed puberty in boys [59]. Conversely, Ostensen $M$. et al. did not demonstrate significant differences between cases and controls in age of menarche, but they reported a reduced fecundity and increased incidence of gynecological disorders in 24 women with diagnosis of JIA in childhood [62]. Alike, other authors did not highlight differences in age of menarche and in adult height between 43 JIA girl and the 59 healthy controls [63].

Abnormalities in puberty may compromise the achievement of peak bone mass (PBM), lean mass and linear growth and may also lead to a psychosocial distress with lower quality of life in some of these adolescents. For these reasons, the assessment of pubertal status needs to be incorporated into the routine evaluation of adolescents with JIA [56, 64].

\section{Pathogenesis of pubertal disorders in JIA}

Persistent inflammatory state, GC treatment and altered body composition with cachexia are mainly responsible for the alteration of regulation of $\mathrm{GH}$ and gonadotropins secretion through multiple mechanisms. Actually, each of these factors, may impact in parallel on two aspects of the pubertal process that mutually influence each other: the hypothalamic-pituitary-gonadal axis, on one hand, and skeletal development by effects on the bone and the growth plate locally, on the other [56].

\section{JIA and hypothalamic-pituitary-gonadal axis}

The production of cytokines, in particular TNF- $\alpha$, IL-1 and IL-6, has effects on the gonadotropins axis that are similar and concomitant to those described for those on GH-IGF-1 axis. The understanding of the mechanisms underlying the link between inflammatory cytokinegonadotropin axis is given by few experimental studies of translational medicine. Inflammatory cytokines have been shown to act at the level of the central nervous system (CNS) [65]. IL-1-immunopositive fibers can be found in human and rat hypothalamus and IL-1 receptors are present in the rat brain. The injection of IL-1 $\beta$ in the cerebral ventricles of rats reduces the luteinizing hormone (LH) increase due to inhibition of LH-releasing hormone (LHRH) release [66]. This effect is mediated via IL-1-activation of hypothalamic endogenous opioid peptide system [67]. In rats not only intraventricular injection of IL-1 and TNF- $\alpha$, but also of lipopolysaccharide (LPS) (which triggers secretion of cytokines) blocks the LH-LHRH axis [68]. This has been confirmed by similar studies, which demonstrated that LPS induces a profound transcriptional down-regulation of LHRH receptor gene expression in the anterior pituitary gland throughout the entire estrous rat cycle. While IL-1 $\beta$ appears the most potent inhibitor of the gonadotropin releasing hormone (GnRH)-LH system, IL- $1 \alpha$ and TNF- $\alpha$ are less effective. The IL- 6 role would seem marginal [69]. In addition, kisspeptin-RFamide-related peptide (RFRP) system is another link between inflammation and gonadal function [70]. In fact, kisspeptin-GPR54 signaling, particularly expressed in the hippocampal dentate gyrus, has an important role in initiating $\mathrm{GnRH}$ at puberty; instead, RFRP-3 has been implicated in GnRH neuronal inhibition in mammalians. It has been proposed that the balance between these systems is related to the maintenance of the fine-tune excitability of $\mathrm{GnRH}$ neurons and the timing of the preovulatory LH surge [71]. The injection of TNF- $\alpha$ in primary cultures of human fetal hypothalamic cells of $\mathrm{GnRH}$ neurons reduces GnRH secretion via downregulation of kisspeptin signaling [72]. On the other hand, LPS injection has been demonstrated to increase hypothalamic RFRP mRNA levels in rodents, thus increasing the negative $\mathrm{GnRH}$ LH surge regulation mechanism [73].

The systemic administration of GCs, especially if prolonged and started in the pre-pubertal period, contributes to cause the GnRH-gonadotropin-gonadic axis alteration [74]. Such effects not only occur via centrally inhibition at the level of the hypothalamus and pituitary gland, but it is also the consequence of a reduced secretion of sex steroid from the gonads or impaired organ sensitivity to sex steroids [75]. Both chronic and acute administration of GCs in male rats result in alteration of reproductive axis: chronic use lead to a significant decrease of hypothalamic GnRH mRNA, while short-term GCs treatment is related to follicle stimulating hormone (FSH) $\beta$ mRNA levels decrease [74]. In addition, in vivo studies on adult women and adolescents treated with GCs affected by other rheumatological diseases highlighted a suppression of LH secretion and an upregulation of FSH levels, assuming the possibility of an effect on the gonadal function $[76,77]$.

\section{JIA and bone}

The achievement of about $50 \%$ of the PBM is reached during puberty because of an increase in bone cortical thickness and a stimulus for trabecular mineralization, in parallel with an increase in growth plate enlargement $[56,78,79]$. Low PBM may result from clinical 
conditions associated with pubertal abnormalities or be directly a consequence of delayed puberty, highlighting the link between puberty and bone development [79-81].

The international study "The Bone Mineral Density in Childhood Study" demonstrated that the age at onset of puberty is a strong negative predictor of BMC and BMD in all DXA skeletal site in adulthood [82]. JIA patients with delayed puberty (34\%) had significantly decreased BMD z-score values, compared to controls; in addition, the age of menarche seemed to be related to peripubertal mineralization [83]. In JIA children, many overlapping mechanisms are responsible for the uncoupling of bone formation from bone reabsorption [83-86]. The reduction of muscle mass, caused by malnutrition and physical immobility, reduces the force on bone load lines, which is an important stimulus for the deposition of bone matrix. Chronic GC administration may lead to a negative calcium balance, via the decrease of intestinal calcium absorption and the increase of renal tubular urinary calcium excretion $[85,86]$. Pro-inflammatory cytokines (mainly TNF- $\alpha$ ), the excess of GCs as well as the reduced gonadal hormonal stimulation act on the nuclear factor kappa $\beta$ (NF-KB) ligand (RANKL)/RANK/ osteoprotegerin (OPG) system and alter the balance between osteoclastic apoptosis and osteoblastic matrix formation. Moreover, some of the effects on peripheral bone reabsorption of the gonadal axis, mainly mediated by estradiol, seems to be mediated indirectly by IGF-1 [86]. Recently WNT signaling pathway and the WNT soluble antagonist dickkopf-1 (DKK1) appeared to be an additional mechanism. Secretion of DKK1 by the synovia is stimulated by inflammatory status and suppresses bone deposition acting on osteoblast differentiation [85]. Therefore, pubertal delay is an additional insult to the bone accrual children with JIA, in addition to inflammatory cytokines, GCs use, low physical activity and malnutrition, with the risk of osteoporosis [56]. The osteoporosis in children has been defined by "Official Positions of the International Society for Clinical Densitometry (ISCD)" published in 2013 as the presence of one or more vertebral fractures (VF) in the absence of local disease or high-energy trauma or Z-score of BMD or BMC $\leq$ -2 (adjusted for size in cases of children measuring below the 3rd percentile) and a history of clinically significant fractures (two or more long bone fractures occurring by age of 10 years or three or more long bone fractures at any age up to age 19 years) [87]. As well as a reduction in BMD, evaluated by DXA, the presence of mostly asymptomatic VF has been found in more than $10 \%$ of JIA patients, especially with systemic and polyarticular onset [88]. This condition must be taken into account in the follow-up of children with JIA [89]. Fig. 2 illustrates the main effects of inflammation on GnRH-gonadotropingonadic axis and bone dysfunction.

\section{Management and therapy of pubertal disorders in JIA}

Monitoring of puberty and bone health is essential for a complete health assessment of adolescents with JIA. In children with chronic inflammatory diseases such as JIA, an assessment of the pubertal stage through the Tanner scale every 6 months from the age of 9 years is recommended. In particular, the failure to initiate puberty over the age of 14 in boys and 13 years in girl should lead the pediatric rheumatologist to consult the pediatric endocrinologist in order to assess the need for induction of puberty [56]. Linear growth should be always evaluated at each medical examination: a pubertal delay, which can parallel growth retardation, can be recovered in many patients upon the disease remission [56, 79]. Evaluation of a wrist $\mathrm{x}$-ray for bone age could reveal a constitutional delay in growth and puberty, which can be recovered once good disease control is achieved. Indeed, an appropriate disease control and the induction of clinical remission is the main goal to be achieved in children with JIA and pubertal disorder. Induction of puberty with sex steroid must be timely weighted together with the pediatric endocrinologist, identifying properly when the use of drugs acting on growth and the cytokine inflammatory activity are minimized. It is in order to allow adequate development of secondary sexual characteristics, evaluating potential for linear growth and the need to optimize bone mass accrual. In the medical literature there are no studies evaluating specific therapeutic sex steroid regimens for patients with JIA and pubertal delay. The use of standard therapeutic regimens offers multiple options such as intramuscular medroxyprogesterone acetate, transdermal $17-\beta$-oestradiol patches or oral ethinyl estradiol or $17-\beta$-oestradiol for girl and intramuscular testosterone enanthate orcypionate, propionate, transdermal testosterone $1 \%$ gel or oral testosterone undecanoate for boys [56].

Close monitoring of bone accrual is essential in JIA patients. Recently, the "Osteogenesis Imperfecta and Childhood Osteoporosis Working Group of the Spanish Society of Pediatric Rheumatology" published the guidelines for early diagnosis and treatment of osteporosis secondary to chronic conditions, including JIA [89]. Adequate nutritional vitamin $\mathrm{D}$ and calcium intake, exposure to sunlight on the hands, face and arms for6-8 $\mathrm{min} /$ day in the summer and $30 \mathrm{~min} /$ day during the coldest months of the year and adequate treatment of the disease represent the cornerstones for the optimization of bone health in JIA patients. A lumbar spine and total body less head (TBLH) DXA are recommended in JIA patients younger than 6 years in the presence of fragility fractures and in JIA patient older than 6 years if not presenting rapid remission of disease in treatment with GCs. In patients treated with GCs a lateral spine x-ray is 


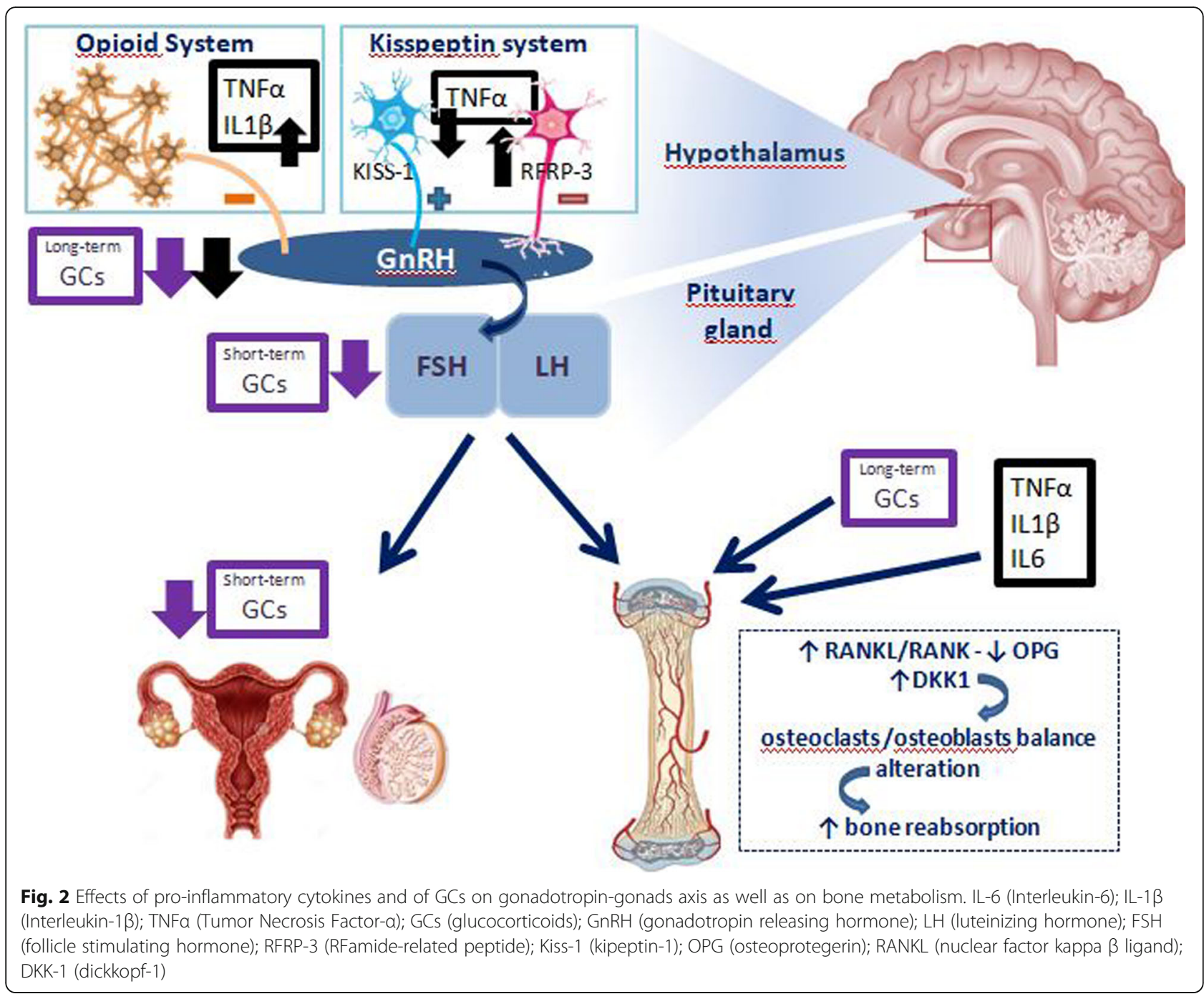

recommended at the beginning (within 6 months) of treatment and after 1 year, then every 9 to 12 months if treatment continues [89]. In patients who will receive GCs for 3 months or more, it is wise to give calcium supplementation or optimization of calcium (500-1000 $\mathrm{mg} /$ day) and vitamin D intake (400 IU/day), maintained for 3 months after discontinuation of GCs. Treatment with bisphosphonate (BP) is indicated in children with osteoporosis, according to ISCD 2013 position criteria, preferring the intravenous administration if a VF is present and oral treatment is in the decalage phase. The main important BPs used in children are shown in Table $1[89,90]$. In addition, in early puberty (Tanner 2), the use of BPs presents additional indications, due to the importance of this phase in achieving the PMB. Indeed, BPs use can be considered, for pubertal patients with risk factors, or with BMD z-score $\leq-2.5 \mathrm{SD}$ with a declining trajectory confirmed at least on two separate occasions with 1 year apart, or a BMDZ score $\leq-3 \mathrm{DS}$ with a declining trajectory confirmed on at least on two separate occasions with 1 year apart, even in the absence of osteoporosis [89].

\section{Delayed puberty and growth impairment in patients with JIA: a close relationship}

Delay of puberty in patients with chronic diseases may result in a decrease in duration and intensity of the pubertal growth spurt resulting in a loss of final height. Some authors reported that total pubertal growth (TBG) in 64 peri-pubertal JIA patients treated with GH $(0.33$ $\mathrm{mg} / \mathrm{kg} /$ week) was increased by a factor of 1.5 greater in comparison to controls, with a gain of +1.16 in height SD during puberty, leading to a significantly better final height [91]. In JIA patients treated with GH, mean TPG was $23.2 \mathrm{~cm}$ in boys and $19.8 \mathrm{~cm}$ in girls, lower than healthy children $(20-35 \mathrm{~cm})$, but significantly higher than controls $(15 \mathrm{~cm}$ in boys and $12.5 \mathrm{~cm}$ in girls). Moreover, for each increase in age at puberty onset by 1 
Table 1 Main bisphosphonates used in pediatric age (adapted from Baroncelli G.I. et al.) [90]

\begin{tabular}{|c|c|c|c|c|}
\hline Name & Oral & Parenteral & Dose & Relative potency \\
\hline Etidronate & + & + & $\begin{array}{l}\text { p.o.:5-40 mg/kg per day } \\
\text { iv: } 400 \mathrm{mg} \text { per day per } 2 \text { weeks, every } 3 \text { months }\end{array}$ & 1 \\
\hline Clodronate & + & + & $\begin{array}{l}\text { p.o.: } 200 \mathrm{mg} \text { per day /subdivided into } 3 \text { doses } \\
\text { iv: (dilute in } 200-250 \mathrm{ml} \text { NSS, in } 2-3 \mathrm{~h} \text { ) } 2 \mathrm{mg} / \mathrm{kg} \text { per day every } 3-6 \text { months }\end{array}$ & 10 \\
\hline Pamidronate & & + & (dilute in 100-250 ml of NSS, in 3-4 h)0.5-1.5 mg/kg per day for 3 days; every 2-6 months) & 100 \\
\hline Neridronate & & + & (dilute in 200-250 ml NSS, in 3 h) 1-2 mg/kg per every 3-6 months & 100 \\
\hline Zoledronate & & + & (dilute in $50 \mathrm{ml} \mathrm{NSS}$, in 30-45 min) $0.015-0.05 \mathrm{mg} / \mathrm{kg}$ every $3-6$ months & $>10,000$ \\
\hline Aledronate & + & & $\begin{array}{l}1-2 \mathrm{mg} / \mathrm{kg} / \text { week } \\
<40 \mathrm{~kg}: 5 \mathrm{mg} / \text { day or } 35 \mathrm{mg} / \text { week } \\
\text { > } 40 \mathrm{~kg}: 10 \mathrm{mg} / \text { day or } 70 \mathrm{mg} / \text { week } \\
\text { Maximum dose: } 70 \mathrm{mg} / \text { week }\end{array}$ & $100-1000$ \\
\hline Risedronate & + & & $\begin{array}{l}15 \mathrm{mg} \text { per week }(<40 \mathrm{~kg}) \\
30 \mathrm{mg} \text { per week }(>40 \mathrm{~kg}) \\
\text { Maximum dose: } 30 \mathrm{mg} / \text { week }\end{array}$ & $1000-10,000$ \\
\hline
\end{tabular}

Abbreviations: iv intravenous, p.o. per os, NSS normal saline solution

year, height gain was reduced $2.3 \mathrm{~cm}$ in $\mathrm{GH}$ treated patients and by $4.2 \mathrm{~cm}$ in controls during puberty, showing that GH could in part counteract the negative effect of pubertal delay on final height. This fits the suggestion to reduce the height deficit of short JIA patients before the onset of puberty. Some authors presented a long-term evidence (up to 10 years) that the adult height of sJIA patients can be normalized with an hormonal combination strategy, by postponing pubertal onset with a gonadotropin-releasing hormone analog until a more normal late-prepubertal height is reached or until the potential of prepubertal growth is exhausted and then promoting growth with high dose $\mathrm{rhGH}(0.5 \mathrm{mg} / \mathrm{kg} /$ week on average) when inflammation is reduced and high GCs doses are not needed [92]. In children with JIA, the reduction of $\mathrm{GC}$ doses and the initiation of a biological therapy favor an increment in height velocity, which does not evolve in a frank catch-up growth [93, 94]. On the contrary, if rhGH is added at high dose in a low-inflammatory condition, it is able to favor a prepubertal growth acceleration, comparable with the catch-up growth response in GH-deficient patients. This is also because this particular moment takes advantage of the period of relative glucocorticoid deficiency following prolonged steroid therapy, which is favorable to height gain [95].

\section{Conclusions}

In patients with JIA, growth impairment and pubertal delay are well-known long-term complications. In the active phases of the disease, patients with polyarticular and sJIA show consistent growth impairment, while some children do experience "catch-up" growth following reduction in disease activity or reduction of GCs doses. Although new therapeutic options are available, there are still $10-20 \%$ of patients with severe forms of the disease who show continuous growth impairment, ending in a short final stature. Pubertal delay in patients with JIA can be a resource, as it can allow the adolescent to exploit the growth potential reserve, upon reaching the disease remission or the lowest disease activity. On the other hand, delayed puberty is associated with a reduction in the PBM with the possibility of concomitant or future bone fragility. Moreover, the effect of GH therapy is greater if started in patients with controlled disease, steroid-sparing therapy and pre-pubertal stage. Therefore, the clinician's goal is to take advantage of patient's linear growth potential and simultaneously optimize bone accrual and health. It is advisable that rhGH therapy should be a "target therapy", tailored to the patient and based on pubertal stage, bone age, degree of systemic inflammation and ongoing treatment.

\section{Abbreviations}

JIA: Juvenile idiopathic arthritis; ILAR: International League of Associations for Rheumatology Classification; sJIA: Systemic juvenile idiopathic arthritis; RF: Rheumatoid Factor; PsA: Psoriasic arthritis; GCs: Glucocorticoids; CAPS: Childhood arthritis prospective study; CHAQ: Childhood health assessment questionnaire; BMI: Body mass index; ReACCh-Out: Research in Arthritis in Canadian Children emphasizing Outcomes; GH: Growth hormone; IGFs: Insulin-like growth factors; GHRs: Growth hormone receptors; PI3K: Phosphatidyl-inositol-3-kinase; MAPK: Mitogen-activated protein kinase; IRSs: Insulin receptor substrates; PKC: Protein Kinase C; STAT: Signal transducers and activators of transcription; GHBP: Growth hormone binding protein; IGFBPs: Insulin-like growth factor binding proteins; ALS: Acid labile subunit; ILs: Interleukins; IFN: Interferon; IL-6: Interleukin-6; IL-1ß: Interleukin$1 \beta$; TNF-a: Tumor necrosis factor-a; NSE: Neuro-specific enolase; JAK: Janus kinase; COMP: Cartilage oligomeric matrix protein; BAP: Bone alkaline phosphatase; MMP: Metalloproteinases; SOCS: Suppressor of cytokine signaling; HSDS/CA: Height standard deviation score for chronological age; HV: Height velocity; FGF-21: Fibroblast growth factor-21; LEPROT: Leptin receptor overlapping transcript; LEPROTL1: Leptin receptor overlapping transcript-like 1; ETN: Etanercept; JADAS-71: Juvenile arthritis disease activity score-71; MDA: Minimal disease activity; rhGH: Recombinant human growth hormone; BMD: Bone mineral density; OGTT: Oral glucose tolerance testing; RCT: Randomized control trials; CSA: Cross-sectional area; PBM: Peak bone mass; CNS: Central Nervous System; LH: Luteinizing hormone; LHRH: LHreleasing hormone; $\mathrm{GnRH}$ : Gonadotropin releasing hormone; FSH: Follicle 
stimulating hormone; LPS: Lipopolysaccharide; RFRP: RFamide-related Peptide; OPG: Osteoprotegerin; BMC: Bone mineral content; NF-KB: Nuclear factor kappa $\beta$; ISCD: International Society for Clinical Densitometry; VF: Vertebral fractures; TBLH: Total body less head; BP: Bisphosphonate; TPG: Total pubertal growth

\section{Acknowledgements}

Not applicable.

\section{Authors' contributions}

$\mathrm{dDM}, \mathrm{DG}$ and $\mathrm{BL}$ wrote the manuscript. CF reviewed the manuscript. The author(s) read and approved the final manuscript.

\section{Funding}

Not applicable.

\section{Availability of data and materials} Not applicable.

\section{Declarations}

\section{Ethics approval and consent to participate}

Not applicable.

\section{Consent for publication}

Not applicable.

\section{Competing interests}

The authors declare that they have no competing interests.

Received: 11 August 2020 Accepted: 4 March 2021

Published online: 12 March 2021

\section{References}

1. Ravelli A, Martini A. Juvenile Idiopathic Arthritis. Lancet. 2007;369(9563):767-78.

2. Prakken B, Albani S, et al. Juvenile Idiopathic Arthritis. Lancet. 2011; 377(9783):2138-49.

3. Petty RE, Southwood TR, et al. International League of Associations for Rheumatology classification of juvenile idiopathic arthritis: second revision Edmonton, 2001. J Rheumatol. 2004 Feb;31(2):390-2.

4. Simon D, Fernando C, et al. Linear Growth and Final Height in Patients With Systemic Juvenile Idiopathic Arthritis Treated With Longterm Glucocorticoids. J Rheumatol. 2002 Jun;29(6):1296-300.

5. MacRae VE, Wong SC, et al. Cytokine Actions in Growth Disorders Associated With Pediatric Chronic Inflammatory Diseases (Review). Int J Mol Med. 2006;18(6):1011-8.

6. McErlane F, Carrasco R, et al. Growth patterns in early juvenile idiopathic arthritis: Results from the Childhood Arthritis Prospective Study (CAPS) Semin Arthritis Rheum. 2018;48(1):53-60.

7. Guzman J, Kerr T, et al. Growth and Weight Gain in Children With Juvenile Idiopathic Arthritis: Results From the ReACCh-Out Cohort. Pediatr Rheumatol Online J. 2017;15(1):68.

8. Nilsson O, Marino R, et al. Endocine regulation of the growth plate. Horm Res. 2005;64(4):157-65.

9. Elis $\mathrm{S}, \mathrm{Wu} Y$, et al. Unbound (biovailable) IGF1 enhances somatic growth. Dis Model Mech. 2011:4:649-58.

10. Wong SC, Dobie R, et al. Growth and the Growth Hormone-Insulin Like Growth Factor 1 Axis in Children With Chronic Inflammation: Current Evidence, Gaps in Knowledge, and Future Directions. Endocr Rev. 2016 Feb; 37(1):62-110.

11. Gaspari S, Marcovecchio ML, et al. Growth in juvenile idiopathic arthritis: the role of inflammation. Clin Exp Rheumatol. 2011;29:104-10.

12. Cirillo F, Lazzeroni $P$, et al. Inflammatory diseases and growth: effects on the GH-IGF axis and on growth plate. Int J Mol Sci. 2017 Aug. 1878;18(9):31.

13. MacRae VE, Wong SC, et al. Cytokine profiling and in vitro studies of murine bone growth using biological fluids from children with juvenile idiopathic arthritis. Clin Endocrinol (Oxf). 2007 Sep;67(3):442-8.

14. De Benedetti F, Meazza C, et al. Effect of IL-6 on IGF binding protein-3: a study in IL-6 transgenic mice and in patients with systemic juvenile idiopathic arthritis. Endocrinology. 2001;142:4818-26.
15. Farquharson C, Ahmed SF. Inflammation and linear bone growth: the inhibitory role of SOCS2 on GH/IGF1 signaling. Pediatr Nephrol. 2013;28: 547-56.

16. Choukair D, Hugel U, et al. Inhibition of IGF1-related intracellular signaling pathways by proinflammatory cytokines in growth plate chondrocytes. Pediatr Res. 2014:76:245-51.

17. Broussard SR, McCusker RH, et al. IL-1 $\beta$ impairs insulin-like growth factor 1 induced differentiation and downstream activation signals of the insulin-like growth factor I receptors in myoblasts. J Immunol. 2004;172:7713-20.

18. Lang $\mathrm{CH}$, Fan J, et al. IL-1 receptor antagonist attenuates sepsis-indiced alterations in the IGF system and protein synthesis. Am J Physiol Endoc. 1996;M270:E430-7.

19. MacRae VE, Farquharson $C$, et al. The restricted potential of growth plate chondrogenesis and longitudinal bone growth following exposure to proinflammatory cytokines. J Endocrinol. 2006;189:319-28.

20. Legendre F, Dudhia J, et al. JAK/STAT but not ERK1/ERK2 pathway mediates interleukin (IL)-6/soluble IL-6R down regulation of type II collagen, aggrecan core, and link protein transcription in articular chondrocytes. Association with a down-regulation of Sox-9 expression. J Biol Chem. 2003;278:2903-12.

21. De Benedetti F, Rucci $N$, et al. Impaired skeletal development in interleukin6-transgenic mice: a model for the impact of chronic inflammation on the growing skeletal system. Arthritis Rheum. 2006;54:3551-63.

22. Wehmeyer C, Pap T, et al. The role of stromal cells in inflammatory bone loss. Clin Exp Immunol. 2017;189:1-11.

23. Nakajima $S$, Naruto $T$, et al. Improvement of reduced serum cartilage oligomeric matrix protein levels in systemic juvenile idiopathic arthritis patients treated with the anti-interleukin- 6 receptor monoclonal antibody tocilizumab. Mod Rheumatol. 2009:19:42-6.

24. Murakami S, Lefebvre $V$, et al. Potent inhibition of the master chondrogenic factor Sox9 gene by interleukin-1 and tumor necroisis factor-alpha. J Biol Chem. 2000;275:3687-92.

25. Fujita T, Fukayama R, et al. Dexamethasone inhibits insulin-induced chondrogenesis of ATDC5 cells by preventing PI3K-Akt signaling and DNA binding of Runx2. J Cell Biochem. 2004:93:374-83.

26. Mushtaq T, Farquharson C, et al. Glucocorticoid effects on chondrogenesis, differentiation and apoptosis in the murine ATDC5 chondrocyte cell line. J Endocrinol. 2002;175:705-13.

27. Zaman F, Chrysis D, et al. Dexamethasone differentially regulates BCl-2 family proteins in human proliferative chondrocytes: role of pro-apoptotic Bid. Toxicol Lett. 2014;224:196-200.

28. Wang SJ, Yang YH, et al. Attained adult height in juvenile rheumatoid arthritis with or without corticosteroid treatment. Clin Rheumatol. 2002;21: 363-8.

29. Souza LS, Machado SH, et al. Growth velocity and interleukin 6 concentrations in juvenile idiopathis arthritis. J Rheumatol. 2008:35:2265-71.

30. Rall LC, Roubenoff R. Rheumatoid cachexia: metabolic abnormalities, mechanisms and interventions. Rheumatology (Oxf). 2004;43:1219-23.

31. Wu S, Grunwald T, et al. Increased expression of fibroblast growth factor 21 (FGF21) during chronic undernutrition causes growth hormone insensitivity in chondrocytes by inducing leptin receptor overlapping transcript (LEPROT) and leptin receptor overlapping transcript-like 1 (LEPROTL1) expression. J Biol Chem. 2013;288(38):27375-83.

32. Uettwiller F, Perlbarg J, et al. Effect of biologic treatments on growth in children with juvenile idiopathic arthritis. J Rheumatol. 2014;41(1):128-35.

33. Billiau $A D$, Loop $L$, et al. Etanercept improves linear growth and bone mass acquisition in MTX-resistant polyarticular-course juvenile idiopathic arthritis. Rheumatology. 2010;49:1550-8.

34. Kearsley-Fleet L, Hyrich KL, et al. Growth in Children and Adolescents With Juvenile Idiopathic Arthritis Over 2 Years of Treatment With Etanercept: Results From the British Society for Paediatric and Adolescent Rheumatology Etanercept Cohort Study. Rheumatology (Oxf). 2015 Jul;54(7):1279-85.

35. Tynjala $P$, Lahdenne $P$, et al. Impact of anti-TNF treatment on growth in severe juvenile idiopathic arthritis. Ann Rheum Dis. 2006;65:2265-71.

36. Vojvodich PF, Hansen JB, et al. Etanercept treatment improves longitudinal growth in prepubertal children with juvenile idiopathic arthritis. Rheumatol. 2007:34:2481-5.

37. Minden K. Adult outcomes of patients with juvenile idiopathic arthritis. Horm Res. 2009;72(Suppl 1):20-5.

38. Bechtold S, Ripperger $P$, et al. Growth hormone increases final height in patients with Juvenile Idiopathic Arthritis: data from a randomized controlled study. J Clin Endocrinol Metab. 2007;92:3013-8. 
39. Svantesson $\mathrm{H}$. Treatment of growth failure with human growth hormone in patients with juvenile chronic arthritis. A pilot study. Clin Exp Rheumatol. 1991;9(suppl 6):47-50.

40. Davies UM, Rooney M, et al. Treatment of growth retardation in juvenile chronic arthritis with recombinant human growth hormone. J Rheumatol. 1994:21:153-8.

41. Bechtold S, Ripperger $P$, et al. Bone mass development and bone metabolism in juvenile idiopathic arthritis: treatment with growth hormone for 4 years. J Rheumatol. 2004;31:1407-12.

42. Simon D, Lucidarme N, et al. Effects on Growth and Body Composition of Growth Hormone Treatment in Children With Juvenile Idiopathic Arthritis Requiring Steroid Therapy. J Rheumatol. 2003 Nov;30(11):2492-9.

43. Bismuth $\mathrm{E}$, Chevenne D, et al. Moderate Deterioration in Glucose Tolerance During High-Dose Growth Hormone Therapy in Glucocorticoid-Treated Patients With Juvenile Idiopathic Arthritis. Horm Res Paediatr. 2010;73(6): 465-72.

44. Bechtold S, Ripperger $\mathrm{P}$, et al. GH therapy in juvenile chronic arthritis: results of a two-year controlled study on growth and bone. J Clin Endocrinol Metab. 2001:86:5737-44.

45. Saha MT, Haapasaari J, et al. Growth hormone is effective in the treatment of severe growth retardation in children with juvenile chronic arthritis. Double blind placebo-controlled followup study. J Rheumatol. 2004;31: 1413-7.

46. Bechtold S, Ripperger $P$, et al. Growth hormone improves height in patients with juvenile idiopathic arthritis: 4-year data of a controlled study. J Pediatr. 2003;143:512-9.

47. Simon D, Prieur AM, et al. Early recombinant human growth hormone treatment in glucocorticoid-treated children with juvenile idiopathic arthritis: a 3-year randomized study. J Clin Endocrinol Metab. 2007;92:2567-73.

48. Perez MD, Abrams SA, et al. Effects of rheumatic disease and corticosteroid treatment on calcium metabolism and bone density in children assessed one year after diagnosis, using stable isotopes and dual energy $\mathrm{x}$-ray absorptiometry. J Rheumatol Suppl. 2000;58:38-43.

49. Lien G, Selvaag AM, et al. A two-year prospective controlled study of bone mass and bone turnover in children with early juvenile idiopathic arthritis. Arthritis Rheum. 2005:52(3):833-40.

50. Bechtold S, Ripperger $P$, et al. Dynamics of Body Composition and Bone in Patients With Juvenile Idiopathic Arthritis Treated With Growth Hormone. Clin Endocrinol Metab. 2010;95(1):178-85.

51. Touati G, Prieur AM, et al. Beneficial effects of one-year growth hormone administration to children with juvenile chronic arthritis on chronic steroid therapy. I. Effects on growth velocity and body composition. J Clin Endocrinol Metab. 1998:83:403-9.

52. Simon D. rhGH treatment in corticosteroid-treated patients. Horm Res. 2007 68:38-45.

53. Simon D, Bechtold S. Effects of Growth Hormone Treatment on Growth in Children With Juvenile Idiopathic Arthritis. Horm Res. 2009;72(Suppl 1):55-9.

54. Raivio T, Miettinen PJ. Constitutional delay of puberty versus congenital hypogonadotropic hypogonadism: Genetics, management and updates. Best Pract Res Clin Endocrinol Metab. 2019;33(3):101316.

55. Wood CL, Lane LC, et al. Puberty: Normal physiology (brief overview). Best Pract Res Clin Endocrinol Metab. 2019;33(3):101265.

56. Kao KT, Denker M, et al. Pubertal abnormalities in adolescents with chronic disease. Best Pract Res Clin Endocrinol Metab. 2019;33(3):101275.

57. Elchuri SV, Momen JJ. Disorders of Pubertal Onset. Prim Care. 2020;47(2): 189-216.

58. Rusconi R, Corona F, et al. Age at menarche in juvenile rheumatoid arthritis. J Pediatr Endocrinol Metab. 2003;16(Suppl 2):285-8.

59. Maher SE, Ali FI. Sexual maturation in Egyptian boys and girls with juvenile rheumatoid arthritis. Rheumatol Int. 2013:33(8):2123-6.

60. El Badri D, Rostom S, et al. Sexual maturation in Moroccan patients with juvenile idiopathic arthritis. Rheumatol Int. 2014:34(5):665-8.

61. Palmert MR, Dunkel L. Clinical practice, Delayed puberty. N Engl J Med. 2012:366:443e53

62. Ostensen M, Almberg $K$, et al. Sex, reproduction, and gynecological disease in young adults with a history of juvenile chronic arthritis. J Rheumatol. 2000;27(7):1783-7

63. Machado SH, Xavier RM, et al. Height and sexual maturation in girls with juvenile idiopathic arthritis. J Pediatr (Rio J). 2020;96(1):100-7.

64. Simon D. Puberty in chronically diseased patients. Horm Res. 2002;57(Suppl 2):53-6.
65. Izvolskaia MS, Tillet Y, et al. Disruptions in the hypothalamic-pituitarygonadal axis in rat offspring following prenatal maternal exposure to lipopolysaccharide. Stress. 2016;19(2):198-205.

66. Kalra PS, Sahu A, et al. Interleukin-1 inhibits the ovarian steroid-induced luteinizing hormone surge and release of hypothalamic luteinizing hormone-releasing hormone in rats. Endocrinology. 1990;126(4):2145-52.

67. Kalra PS, Fuentes $M$, et al. Endogenous opioid peptides mediate the interleukin-1-induced inhibition of the release of luteinizing hormone (LH)releasing hormone and LH. Endocrinology. 1990;127(5):2381-6.

68. Rivier C, Vale W. Cytokines act within the brain to inhibit luteinizing hormone secretion and ovulation in the rat. Endocrinology. 1990;127(2): 849-56.

69. Watanobe $H$, Hayakawa Y. Hypothalamic Interleukin-1 $\beta$ and Tumor Necrosis Factor-a, but Not Interleukin-6, Mediate the Endotoxin-Induced Suppression of the Reproductive Axis in Rats. Endocrinology. 2003;144:4868-75.

70. Lee CY, Li SY, et al. Lipopolysaccharide reduces gonadotrophin-releasing hormone $(\mathrm{GnRH})$ gene expression: Role of RFamide-related peptide-3 and kisspeptin. Reprod Fertil Dev. 2019;31:1134-43.

71. Khan AR, Kauffman AS. The role of kisspeptin and RFamide-related peptide3 neurones in the circadian-timed preovulatory luteinising hormone surge. $J$ Neuroendocrinol. 2012;24(1):131-43.

72. Sarchielli E, Comeglio P, et al. Tumor necrosis factor-a impairs kisspeptin signaling in human gonadotropin-releasing hormone primary neurons. Clin Endocrinol Metab. 2017;102:46-56.

73. Iwasa T, Matsuzaki T, et al. Hypothalamic Kiss1 and RFRP gene expressions are changed by a high dose of lipopolysaccharide in female rats. Horm Behav. 2014;66:309-16.

74. Gore AC, Attardi B, et al. Glucocorticoid repression of the reproductive axis: effects on $\mathrm{GnRH}$ and gonadotropin subunit mRNA levels. Mol Cell Endocrinol. 2006:256(1-2):40-8.

75. Tetsuka M, Milne M, et al. Expression of 11-beta-hydroxysteroid dehydrogenase, glucocorticoid receptor, and mineralocorticoid receptor genes in rat ovary. Biol Reprod. 1999;60(2):330e5

76. Sakakura M, Takebe $\mathrm{K}$, et al. Inhibition of luteinizing hormone secretion induced by synthetic LRH by long-term treatment with glucocorticoids in human subjects. J Clin Endocrinol Metab. 1975:40(5):774e9.

77. Aikawa NE, Sallum AM, et al. Menstrual and hormonal alterations in juvenile dermatomyositis. Clin Exp Rheumatol. 2010;28(4):571e5.

78. Hardy R, Cooper M. Bone loss in inflammatory disorders. J Endocrinol. 2009; 2013:309-20.

79. Umławska W, Prusek-Dudkiewicz A. Growth retardation and delayed puberty in children and adolescents with juvenile idiopathic arthritis. Arch Med Sci. 2010:6(1):19-23.

80. Gordon RJ, Gordon CM. Adolescents and Bone Health. Clin Obstet Gynecol. 2020:10:1097.

81. Ziglar S, Hunter TS. The effect of hormonal oral contraception on acquisition of peak bone mineral density of adolescents and young women. J Pharm Pract. 2012;25(3):331-40

82. Gilsanz V, Chalfant J, et al. Age at onset of puberty predicts bone mass in young adulthood. J Pediatr. 2011;158(1):100-105.e1052

83. Alfredo M. Relationship between delayed menarche and bone mineralization in patients affected by juvenile idiopathic arthritis (JIA). J Clin. Dens. 2006:9:341.

84. Hardy RG, Cooper MS. Bone loss in inflammatory disorders. J Endocrinol. 2013:2009:309-20.

85. Zhang Y, Milojevic D. Protecting Bone Health in Pediatric Rheumatic Diseases: Pharmacological Considerations. Paediatr Drugs. 2017;19(3):193-211.

86. Jayasena A, Atapattu N, et al. Treatment of glucocorticoid-induced low bone mineral density in children: a systematic review. Int J Rheum Dis. 2015;18(3):287-93

87. Gordon CM, Leonard MB, et al. 2013 pediatric position development conference: executive summary and reflections. J Clin Densitom. 2014;17(2):219-24.

88. LeBlanc CM, Ma J, et al. Incident Vertebral Fractures and Risk Factors in the First Three Years Following Glucocorticoid Initiation Among Pediatric Patients With Rheumatic Disorders. J Bone Miner Res. 2015;30(9):1667-75.

89. Galindo-Zavala R, Bou-Torrent R, et al. Expert panel consensus recommendations for diagnosis and treatment of secondary osteoporosis in children. Pediatr Rheumatol. 2020;18:20

90. Thornton J, Ashcroft DM, et al. Systematic review of effectiveness of bisphosphonates in treatment of low bone mineral density and fragility fractures in juvenile idiopathic arthritis. Arch Dis Child. 2006;91(9):753-61. 
91. Bechtold S, Beyerlein A, et al. Total Pubertal Growth in Patients With Juvenile Idiopathic Arthritis Treated With Growth Hormone: Analysis of a Single Center. Growth Horm IGF Res. 2012 Oct;22(5):180-5.

92. de Zegher F, Reynaert N, et al. Growth Failure in Children with Systemic Juvenile Idiopathic Arthritis and Prolonged Inflammation despite Treatment with Biologicals: Late Normalization of Height by Combined Hormonal Therapies. Horm Res Paediatr. 2018;90(5):337-43.

93. Yokota S, Imagawa T, et al. Long term safety and effectiveness of the antiinterleukin-6 receptor monoclonal antibody tocilizumab in patients with systemic juvenile idiopathic arthritis in Japan. J Rheumatol. 2014 Apr;41(4): 759-67.

94. De Benedetti F, Brunner $\mathrm{H}$, et al. Catch-up Growth During Tocilizumab Therapy for Systemic Juvenile Idiopathic Arthritis: Results From a Phase III Trial. Arthritis Rheumatol. 2015 Mar;67(3):840-8.

95. Elias $L L$, Huebner A, et al. Tall stature in familial glucocorticoid deficiency. Clin Endocrinol (Oxf). 2000 Oct;53(4):423-30.

\section{Publisher's Note}

Springer Nature remains neutral with regard to jurisdictional claims in published maps and institutional affiliations.

Ready to submit your research? Choose BMC and benefit from:

- fast, convenient online submission

- thorough peer review by experienced researchers in your field

- rapid publication on acceptance

- support for research data, including large and complex data types

- gold Open Access which fosters wider collaboration and increased citations

- maximum visibility for your research: over $100 \mathrm{M}$ website views per year

At BMC, research is always in progress.

Learn more biomedcentral.com/submissions 